\title{
Production Fruitière De Quatre Essences Ligneuses Dans La Forêt De Nassou En Zone Soudanienne Du Bénin
}

\author{
Agbani Onodjè Pierre, MSc., Doctorant-Chercheur \\ Laboratoire d'Ecologie Appliquée, Faculté des Sciences Agronomiques, \\ Université d'Abomey-Calavi, 01 BP 526, Cotonou, Benin \\ Institut de Recherche et d'Expérimentation en Médecine et Pharmacopée \\ Traditionnelle, Centre Béninois de la Recherche Scientifique et de \\ l'Innovation BP 06, Porto-Novo, Bénin \\ Gandji Kisito, MSc., Doctorant-Chercheur \\ Tovissodé Frédéric, MSc., Doctorant-Chercheur \\ Laboratoire de Biomathématiques et d'Estimations Forestières, Faculté des \\ Sciences Agronomiques, Université d'Abomey-Calavi, 04 BP 1525, \\ Cotonou, Bénin \\ Karen Hahn, PhD, Enseignant-Chercheur \\ 3Botanical Institute, J.W. Goethe-University Frankfurt, Siesmayerstr.70, \\ D-60054 Frankfurt am Main, Germany \\ Sinsin Brice, PhD, Enseignant-Chercheur \\ Laboratoire d'Ecologie Appliquée, Faculté des Sciences Agronomiques, \\ Université d'Abomey-Calavi, 01 BP 526, Cotonou, Benin
}

Doi: 10.19044/esj.2017.v13n36p352 URL:http://dx.doi.org/10.19044/esj.2017.v13n36p352

\begin{abstract}
Rural populations are closely dependent on plant resources for their daily needs. Fruit productivity of these species is an indicator of their economic potential and their capacity to regenerate. This is also fundamental to define sustainable management strategies of species. The present study assessed the fruits and seeds productivity of four forest species such as Vitex doniana, Afraegle paniculata, Diospyros mespiliformis and Kigelia africana according to the diameter class of trees. The study was carried out in the Nassou forest in soudanian zone of Benin. 09 to 90 trees belonging to four diameter classes $(10-20 \mathrm{~cm}, 20-30 \mathrm{~cm}, 30-40 \mathrm{~cm}$, and $\geq 40 \mathrm{~cm})$ were sampled for each species. The method used is the counting of fruits fallen under the tree through a periodic collection carried out every five days from the fructification to the end of the cycle and the fall of the last fruit. Seeds were then extracted from fruits. Generalized linear models of Poisson family were
\end{abstract}


used to analyse data. Results revealed that the diameter class on the one hand influence significantly (Prob. $\leq 0.01$ ) the production of fruits of $K$. africana and $V$. doniana, and on the other hand the production of seeds of $A$. paniculata, D. mespiliformis and $V$. doniana. Overall, fruits and seeds production is higher for the biggest tress $(\geq 40 \mathrm{~cm})$. Therefore, the diameter of the four studied species would be very useful for genetic improvement aiming the increasing of their productivity.

Keywords: Annual productivity; fruits; diameter class; forest; Benin.

\section{Résumé}

Les populations rurales dépendent étroitement des ressources végétales pour leurs besoins quotidiens. La productivité fruitière et semencière de ces espèces est un indicateur de leur potentiel économique et de leur capacité à se régénérer. Elle est également capitale pour définir des stratégies de gestion durable des espèces. La présente étude a évalué la production de fruits et de semences de quatre essences forestières que sontVitex doniana, Afraegle paniculata, Diospyros mespiliformis, et Kigelia africana en relation avec la classe de diamètre des individus. L'étude a été réalisée dans la forêt de Nassou en zone soudanienne du Bénin. Un échantillon de 9 à 90 arbres appartenant à quatre classes de diamètre (10-20 $\mathrm{cm}, 20-30 \mathrm{~cm}, 30-40 \mathrm{~cm}$, et $>40 \mathrm{~cm}$ ) a été considéré par espèce. La méthode utilisée dans le cadre de cette étude est le comptage des fruits tombés sous l'arbre à travers un ramassage périodique tous les 5 jours depuis la fructification jusqu'à la fin du cycle et de la chute du dernier fruit. Les graines ont été ensuite extraites des fruits. Les modèles linéaires généralisés de la famille de Poisson ont été utilisés pour l'analyse des données. Les résultats ont révélé que la classe de diamètre influence significativement (Prob. $\leq 0.01$ ) d'une part la production de fruits de $K$. africana et $V$. doniana, et d'autre part la production de semences viables de A. paniculata, $D$. mespiliformis et de $V$. doniana. En général la production de fruits et de semences est plus élevée pour les arbres les plus gros $(\geq 40 \mathrm{~cm})$. Ainsi, le diamètre des espèces étudiées pourrait être très utile pour l'amélioration génétique de celles-ci avec pour objectif l'augmentation de leur productivité.

Mots clés: Productivité annuelle; fruits; classe de diamètre; forêt; Bénin

\section{Introduction}

Au Bénin comme dans beaucoup de pays en Afrique sub-saharienne, les populations rurales dépendent étroitement des ressources végétales pour leurs besoins en alimentation, fourrage, bois énergie, pharmacopée, artisanat et autres produits (Belem et al., 2007 ; Sop et al.,2012). Beaucoup d'espèces 
d'arbres sont intéressantes pour l'alimentation humaine sur les marchés locaux et régionaux africains face aux besoins croissants qui découlent de la pression démographique (Kouyaté et al., 2006). Les auteurs ajoutent que généralement le potentiel de production en fruits de ces espèces n'est pas connu et que cela peut constituer un frein pour leur valorisation.

Vitex doniana Sweet., Afraegle paniculata (Shum \& Thonn.) Engl., Diospyros mespiliformis L., et Kigelia africana (Lam.) Benth., sont des espèces agroforestières retrouvées en zones soudanienne dans différents habitats au Bénin. $V$. doniana et $A$. paniculata sont des légumes feuilles traditionnelles beaucoup consommées au Bénin (Achigan Dako et al., 2010). Les fruits murs de $V$. doniana sont directement consommés par de nombreuses communautés rurales (Ky, 2008). L'importance des fruits de $V$. doniana n'est pas reconnue en raison de l'ignorance de leur valeur nutritive, de leur coût, de leur difficulté de stockage et de leur distribution (Sai, 1997; Vunchi et al., 2011). L'utilisation des racines de A. paniculata contre l'hypertension et l'infertilité (Igoli et al., 2005); contre le paludisme et les maux de ventre (Asase et al., 2005) a été démontré en Afrique de l'Ouest. Les fruits, les racines et l'écorce de $K$. africana sont très utilisés en médicine traditionnelle par beaucoup de peuples africains (Pooley 1993; Maisiri and Gundidza 1999; Saini et al., 2009). En Afrique de l'Ouest plus particulièrement, les racines et les fruits non mûrs de $K$. africana, sont utilisés comme vermifuge et dans le traitement de l'hémorroïde et du rhumatisme (Irvine 1961 ; Saini et al., 2009). D. mespiliformis figure parmi les espèces les plus utilisées au Burkina Faso (Zizka et al., 2015) et en zone soudanienne au Bénin (Vodouhê et al., 2009). Toutes ces études antérieures démontrent l'importance socioéconomique de $V$. doniana, A. paniculata, $K$. africana, et $D$. mespiliformis pour les communautés rurales. Du fait de leur importance socioéconomique, la connaissance de la production fruitière de ces espèces serait utile pour mieux élaborer les stratégies de leur valorisation et de leur conservation. Ces espèces sont également menacées par les méthodes d'exploitation non durables qui ne facilitent pas leur régénération à travers la production des fruits.

La production fruitière des espèces forestières apparaît donc comme un indicateur fiable de leur potentiel économique et de leur capacité à se régénérer. Dans ce cas l'indentification des facteurs et paramètres influençant sa variabilité s'avère important. Des investigations ont été menées sur l'influence des paramètres dendrométriques des arbres et morphologiques des fruits (Snook et al., 2005; Kouyaté et al., 2006; Ouédraogo et al., 2014) sur la productivité de certaines espèces. Les résultats de ces investigations ont révélé l'influence des paramètres dendrométriques des arbres et des paramètres morphologiques des fruits sur la productivité des espèces étudiées. Toutefois ces influences varient d'une espèce à une 
autre. Par exemple, Ouédraogo et al. (2014) ont trouvé que la production de calices de Bombax costatum Pellegr. \& Vuillet était influencée par son diamètre à hauteur de poitrine et le diamètre de son houppier. De plus, la production fruitière de Swietenia macrophylla King était influencée par son diamètre à hauteur de poitrine (Snook et al. 2005).

La présente étude vise principalement à développer les stratégies de conservation de quatre espèces ( $V$. doniana, A. paniculata, D. mespiliformis, et $K$. africana) en zone soudanienne au Bénin à travers l'évaluation des facteurs influençant leur productivité. Dans ce contexte et en se basant sur les résultats précédents, la présente étude vise à tester l'hypothèse selon laquelle plus le diamètre des individus des quatre espèces cibles augmente, plus leur production de fruits et de semences augmente.

\section{Matériel et méthodes}

\section{Milieu d'étude}

L'étude a été réalisée dans la forêt de Nassou, située dans la commune de Ouassa-Péhunco (Figure 1) en zone soudanienne du Bénin. Située entre la haute vallée de l'Alibori à l'Est et celle du Mékrou à l'Ouest dans le Sud-Est du Département de l'Atacora entre $10^{\circ} 03^{\prime}$ et $10^{\circ} 45^{\prime}$ de latitude Nord et $1^{\circ} 45^{\prime}$ et $2^{\circ} 15^{\prime}$ de longitude Est. La forêt de Nassou est une mosaïque de forêt dense sèche, de savane et de jachères.

La gestion de la forêt de Nassou est assurée par l'association communale des Praticiens de la Médecine Traditionnelle appelée en Baatonou "Faaba Yéru » et son bras armé "Tim Gbéru ». Son sens d'organisation, sa puissance et la satisfaction donnée aux populations locales lui confère une notoriété à tel point qu'elle est très sollicitée pour des questions relatives à la gestion des ressources naturelles locales de la Commune de Ouassa-Péhunco. Un des membres de cette association spécialiste dans le traitement des maladies démentielles qui habite proche de cette forêt en assure la garde avec ses collègues du village de Nassou qui abrite la forêt. 


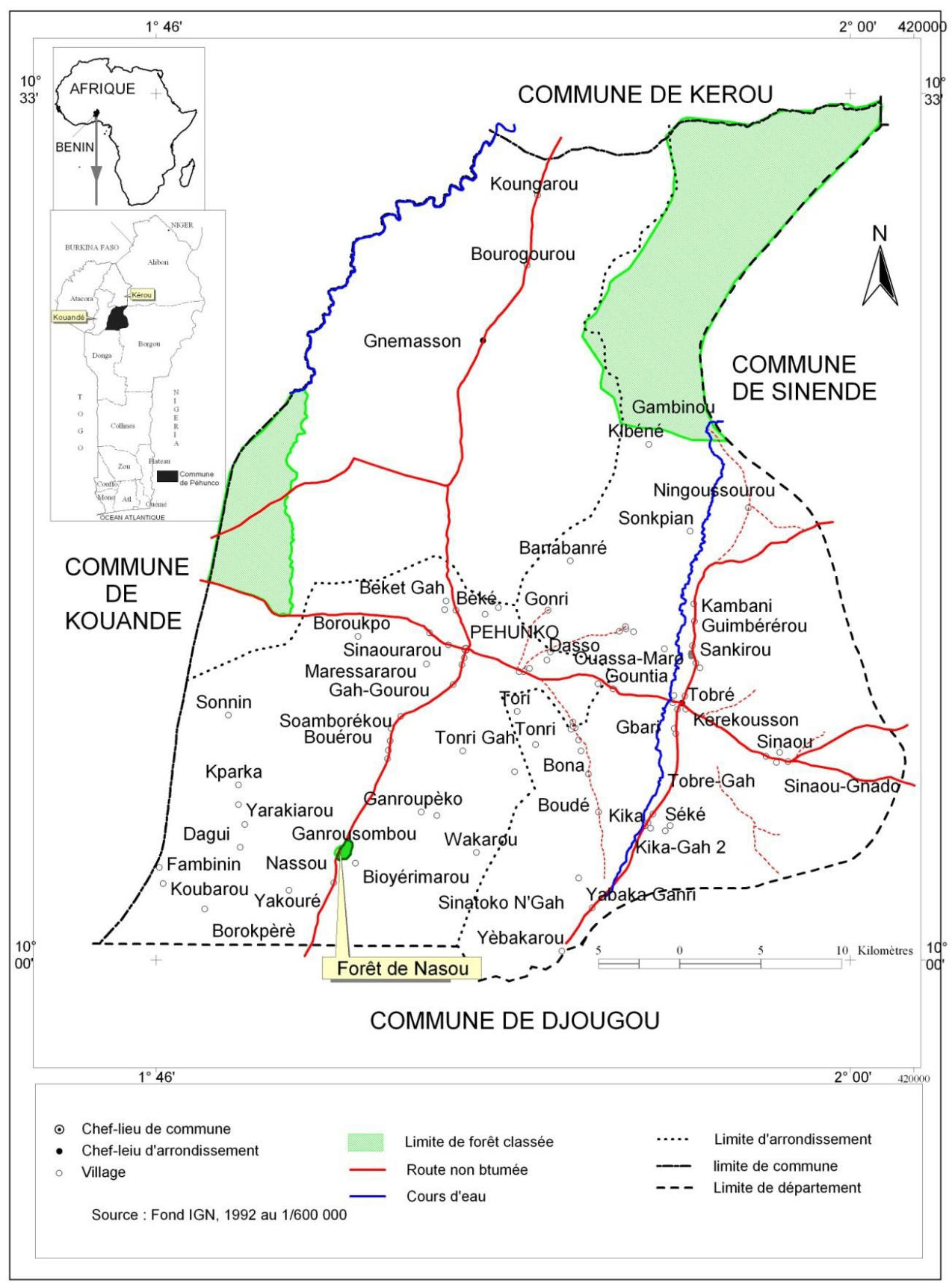

Figure 1 : Localisation de la forêt de Nassou dans la Commune de Ouassa-Péhunco 


\section{Echantillonnage et collecte de données}

Bien que la forêt de Nassou soit une mosaïque de savanes et de forêts denses, un seul individu des quatre espèces, notamment $V$. doniana a été rencontré en savane. En conséquence les données ont été collectées uniquement dans la forêt dense sèche. Quatre classes de diamètre à hauteur d'homme des arbres ont été considérées, notamment $10-20 \mathrm{~cm}, 20-30 \mathrm{~cm}, 30$ $40 \mathrm{~cm}$, et $\geq 40 \mathrm{~cm}$. Le nombre total d'arbres échantillonnés au hasard par espèce était de 98 pour $K$. africana, 80 pour $V$. doniana, 35 pour $D$. mespiliformis et 9 pour A. paniculata. Les faibles nombre d'individus pour les deux dernières espèces sont dus à leur relative rareté dans la forêt. Ces arbres en peuplement naturel ou isolés selon l'espèce, étaient distants d'au moins $100 \mathrm{~m}$. Chacun des individus sélectionné a été marqué et numéroté (voir Photo 1A).

Les fruits de D. mespiliformis sont des drupes composés ou drupéoles ovoïde, jaunâtre à maturité (Hutchinson et Dalziel, 1963; Akoegninou et al., 2006; Arbonnier 2008). Le fruit de $V$. doniana est aussi une drupe mais ellipsoïde et glabre d'environ $3 \mathrm{~cm}$ de long, entouré à la base du calice persistant et agrandi en cupule, noirâtre à maturité, renfermant un noyau dur polyspermé noyé dans une pulpe mince (Hutchinson et Dalziel, 1963; Akoegninou et al., 2006; Arbonnier 2008). Le fruit de $K$. africana est en forme de gros saucisson gris, arrondi aux deux bouts, plus ou moins lisse jusqu'à $90 \mathrm{~cm}$ de long (Hutchinson et Dalziel, 1963; Akoegninou et al., 2006; Arbonnier 2008). Les fruits de A. paniculata sont de très gros drupéoles bacciformes, globuleux à obovoïdes multiséminés, jusqu'à $12 \mathrm{~cm}$ de long à parois lignifiée (4-5mm) à surface vert brillant; habituellement avec 8 loges blanc rosée. Les graines (semences) ovoïdes sont noyées dans un mucilage colloïdal jaune à maturité (Hutchinson et Dalziel, 1963; Akoegninou et al., 2006; Arbonnier 2008).

Il existe plusieurs méthodes d'évaluation de la production des fruits des arbres: le comptage du nombre total de fruits à travers la récolte intégrale des fruits (Cunningham, 2001 ; Ouoba et al., 2006), le comptage des fruits tombés sous l'arbre à travers un ramassage périodique (Lamien et al., 2007), et le comptage par extrapolation (Nacoulma, 2012). La méthode utilisée dans le cadre de cette étude est le comptage des fruits tombés sous l'arbre à travers un ramassage périodique sur toute la saison de production annuelle (Lamien et al., 2007). Le ramassage des fruits tombés est fait tous les 5 jours depuis la fructification jusqu'à la fin du cycle et de la chute du dernier fruit sur le semencier cible. A cet effet, la surface sous houppier de chaque arbre $(+1 \mathrm{~m})$ a été nettoyée (Photo $1 \mathrm{~A} \& \mathrm{~B}$ ) pour faciliter le repérage et le comptage des fruits. 


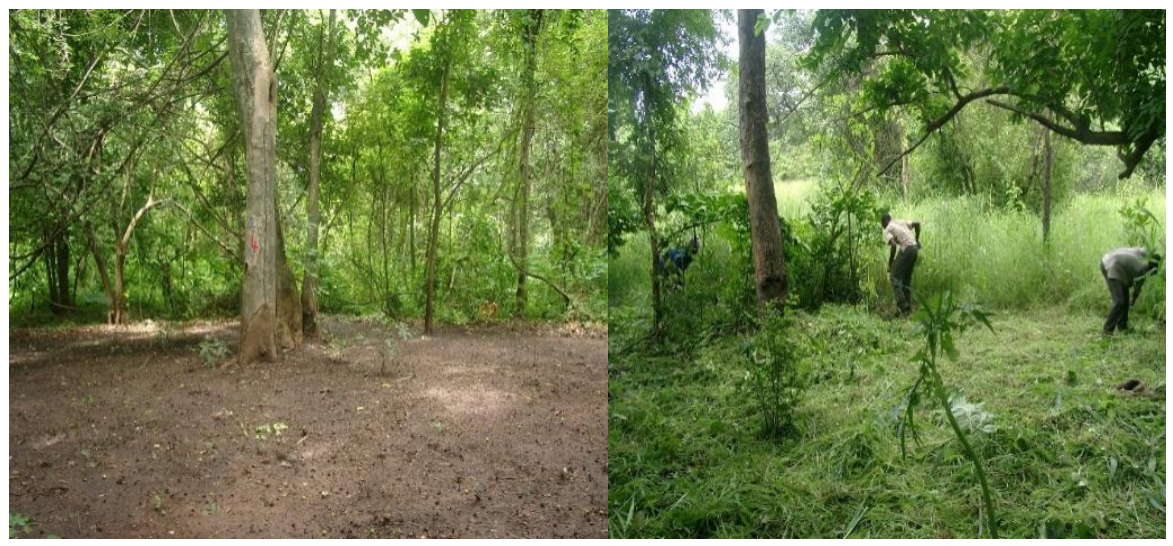

Photo 1. Parterre nettoyé du houppier d'un arbre échantillon (A), Nettoyage du parterre du houppier d'un arbre échantillon (B)
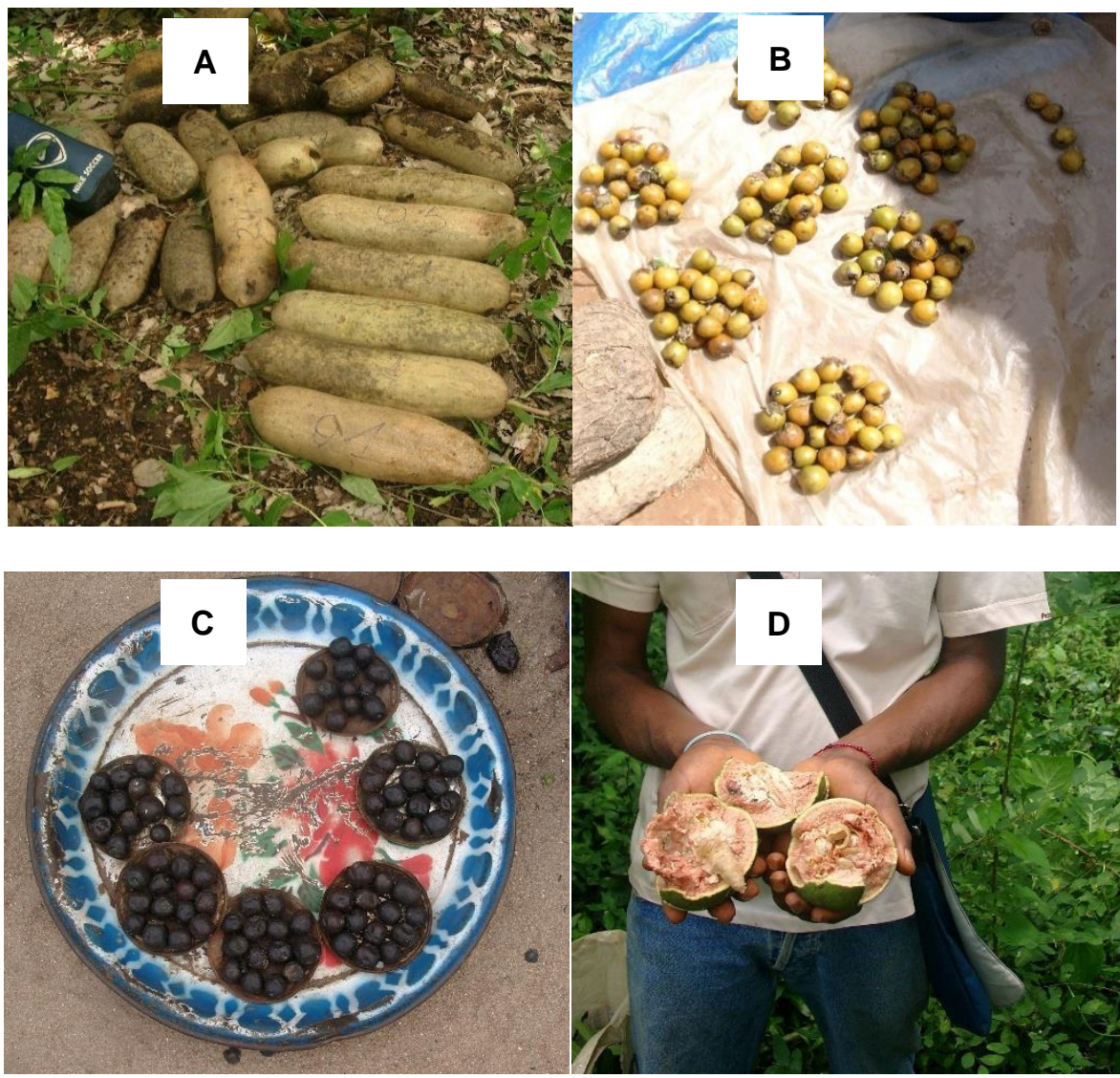

Photo 2. Fruits de K. africana (A), D. mespiliformis (B), V. doniana (C), A. paniculata (D)

Pour chaque arbre, la fructification a été suivie durant toute la période de maturation et de chute des fruits. Les fruits tombés au sol sous chaque 
semencier (fruits intacts sur le semencier, fruits consommés en partie et/ou en minéralisation, fruits verts murs) ont été sommés pour déterminer le nombre total de fruits par arbre. Les graines de D. mespiliformis sont extraites par simples pression du fruit puis comptées. La même méthode a été utilisée pour $V$. doniana. Les fruits de $K$. africana sont fendus à l'aide d'une scie pour accélérer le dessèchement ou la putréfaction rapide de la masse fibreuse par immersion aqueuse afin de facilement extraire les graines (semences) de la masse fibreuse pour le comptage direct (Cunningham, 2001). Quant à A. paniculata, le nombre de graines contenues dans chaque fruit a été déterminé après la cassure de ceux-ci pour évaluer le nombre de graines par fruit. Les graines viables ont été identifiées en utilisant le principe de l'immersion aqueuse. L'équipe de comptage était constituée de 5 personnes. Les dénombrements des fruits et des graines ont été effectués par au moins deux membres de l'équipe pour éviter les erreurs de dénombrement.

\section{Analyses statistiques}

Pour évaluer l'influence de la classe de diamètre des arbres (considérés comme variables explicatives) sur la production annuelle de fruits et de graines par arbre (considérées comme variables expliquées/réponses) pour chaque espèce, des modèles linéaires généralisés ont été utilisés. A cet effet, les distributions de Poisson, quasi-Poisson et binomiale négative ont d'abord été ajustées à chacune des variables réponses (nombre de fruits, nombre de graines). La distribution qui s'ajustait mieux aux données a donc été retenue dans l'élaboration des modèles. Les quartiles (premier, deuxième et troisième) ont été calculés pour mieux décrire les tendances observées. Toutes les analyses statistiques ont été réalisées dans le logiciel R (R Core Development Team, 2013) et le seuil de signification a été fixé à $\alpha=0,05$.Les packages MASS (Venables et Ripley, 2002) et fmsb (Nakazawa, 2014) ont été utilisés pour les modèles linéaires généralisés.

\section{Résultats}

\section{Afraegle paniculata}

A. paniculata a été retrouvé seulement en forêt dense. De même, les individus rencontrés en fructification avaient plus de $40 \mathrm{~cm}$ de diamètre. En conséquence une seule classe de diamètre a été considérée pour cette espèce (Tableau 1). Le nombre total de fruits de A. paniculata collectés par arbre a varié de 18 à 74 fruits pour une moyenne $(\mathrm{m} \pm \mathrm{cv} \%)$ de 45,63 \pm 48,54\% fruits par arbre. La majorité des arbres (75\%) avaient produit au moins 32 fruits par an ( $1^{\mathrm{er}}$ quartile).

La production de graines viables de $A$. paniculata a varié de zéro à

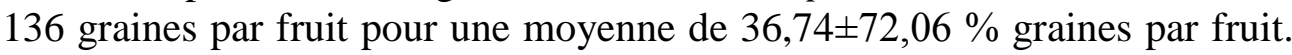


La majorité des fruits comptait au moins 18 graines viables ( $1^{\mathrm{er}}$ quartile) et le quart des fruits contenaient plus de 48 graines ( $3{ }^{\text {ème }}$ quartile) (Figure 2). Le nombre total de graines produit par pied était en moyenne $1182,00 \pm 8,26 \%$ graines/arbre (Tableau 2).

\section{Diospyros mespiliformis}

D. mespiliformis a été retrouvé également seulement en forêt dense. Le nombre total de fruits de $D$. mespiliformis collectés par arbre a varié de 828 à 54590 fruits pour une moyenne $(\mathrm{m} \pm \mathrm{cv} \%)$ de $11118,30 \pm 129,13 \%$ fruits par arbre. Aucune différence significative n'a été observée entre classes de diamètre (régression binomiale négative, Tableau 1). Le nombre moyen de fruits produits par arbre était de 7882,00 $\pm 100,95 \%$ pour les arbres de classe10 à $20 \mathrm{~cm}$, de $9421,3 \pm 46,47 \%$ pour les arbres de 20 à 30 $\mathrm{cm}$ de diamètre, de $13663,00 \pm 84,45 \%$ pour les arbres de 30 à $40 \mathrm{~cm}$ de diamètre et de $10181,4 \pm 66,34 \%$ fruits pour les arbres de plus de $40 \mathrm{~cm}$ de diamètre (Tableau 2). La classe de diamètre de 30 à $40 \mathrm{~cm}$ serait une classe optimale de production fruitière.

La production de graines viables par pied de $D$. mespiliformis variait de zéro à six graines par fruit pour une moyenne de 2,92 $\pm 40,71 \%$ graines par fruit. La majorité des fruits comptait au moins deux graines viables $\left(1^{\mathrm{er}}\right.$ quartile) et le quart des fruits contenaient au moins quatre graines ( $3{ }^{\text {ème }}$ quartile) (Figure 2). Toutefois, le nombre de graines produites par pied de $D$. mespiliformis a varié significativement suivant la classe de diamètre des arbres (régression binomiale négative, Tableau 1). La production de graines était en moyenne de $510420,00 \pm 117,36 \%$ graines pour les arbres de plus de $40 \mathrm{~cm}$ de diamètre, et 14472,00 $\pm 50,14 \%$ graines par arbre de 10 à $20 \mathrm{~cm}$ de diamètre (Tableau 2). En général, le nombre de graines augmentent avec la grosseur des arbres.

\section{Kigelia africana}

$K$. africana a été retrouvé seulement en forêt dense. Le nombre total de fruits de $K$. africana collectés par arbre variait de zéro à 65 pour une moyenne $(\mathrm{m} \pm \mathrm{cv} \%)$ de 7,67 $\pm 159,20 \%$ fruits par arbre. Près du tiers des arbres $(31,25 \%)$ n'avait produit aucun fruit. La classe de diamètre des pieds de $K$. africana a influencé significativement leur production fruitière (régression binomiale négative, Tableau 1). En effet, plus grand était le diamètre de l'arbre, plus élevée était la production fruitière. Ainsi, la production variait d'environ $0,88 \pm 240,02 \%$ fruits/arbre en moyenne pour les pieds de 10 à $20 \mathrm{~cm}$ de diamètre, à 15,00 $\pm 133,00 \%$ fruits/arbre pour les pieds de plus de $40 \mathrm{~cm}$ de diamètre (Tableau 2).

Le nombre de graines viables par fruit de $K$. africana a largement varié, de 25 à 892 graines pour une moyenne $(\mathrm{m} \pm \mathrm{cv} \%)$ de 372,09 \pm 43,60 
graines/fruit. Trois quart des fruits comptait au moins 252 graines viables $\left(1^{\mathrm{er}}\right.$ quartile) et le quart des fruits contenait au moins 481 graines viables (Figure 2). Le nombre de graines viables produites par pied de $K$. africana n'a pas significativement varié suivant la classe de diamètre des arbres (régression binomiale négative, Tableau 1). La production de graine a toutefois numériquement fluctuée entre les classes de diamètre, de 346,75 $\pm 222,90 \%$ graines en moyenne par arbre de 10 à $20 \mathrm{~cm}$ de diamètre à 5774,11 $\pm 168,03$ $\%$ graines pour les arbres de plus de $40 \mathrm{~cm}$ de diamètre (Tableau 2). Mais la tendance n'était pas monotone, les arbres de 30 à $40 \mathrm{~cm}$ de diamètre ayant produit en moyenne $2857,38 \pm 117,52 \%$ graine/arbre alors que les arbres de 20 à $30 \mathrm{~cm}$ de diamètre (moins gros) avaient produit en moyenne 3458,29 \pm $108,43 \%$ graine/arbre (plus de graines).

\section{Vitex doniana}

Vitex doniana a été également retrouvé en forêt dense. Le nombre total de fruits de $V$. doniana collectés par arbre a varié de 580 à 11955 fruits pour une moyenne $(\mathrm{m} \pm \mathrm{cv} \%)$ de $3881,64 \pm 91,63 \%$ fruits par arbre. La production fruitière par arbre a varié significativement suivant la classe de diamètre (régression binomiale négative, Tableau 1).

La production de graines viables par $V$. doniana a varié de un à quatre graines par fruit pour une moyenne de $1,17 \pm 43,13 \%$ graines par fruit. La majorité des fruits $(88,03 \%)$ comptait une graine viable (Figure 2). La production de graines par pied de $V$. doniana a varié significativement suivant la classe de diamètre (régression binomiale négative, Tableau 2).

Tableau 1. Variations de la production fruitière des espèces suivant la classe de diamètre : régression binomiale négative

\begin{tabular}{|c|c|c|c|}
\hline Espèces & Statistiques & Production fruitière & Production semencière \\
\hline \multirow{4}{*}{ A. paniculata } & DDL & 3 & 3 \\
\cline { 2 - 4 } & RD & 8,32 & 8,18 \\
\cline { 2 - 4 } & Prob. & $0,162^{\text {ns }}$ & $0,002^{* *}$ \\
\hline \multirow{3}{*}{ D. mespiliformis } & & & 3 \\
\cline { 2 - 4 } & DDL & 3 & 23,69 \\
\cline { 2 - 4 } & RD & 17,23 & $0,006^{* *}$ \\
\hline \multirow{3}{*}{ K. africana } & Prob. & $0,233^{\text {ns }}$ & 3 \\
\hline & & & 36,53 \\
\cline { 2 - 4 } & DDL & 3 & $0,213^{\text {ns }}$ \\
\cline { 2 - 4 } & RD & 35,16 & 3 \\
\hline Prob. & & $0,002^{* *}$ & 29,53 \\
\hline & DDL & & $0,007^{* *}$ \\
\cline { 2 - 4 } & RD & 29,40 & \\
\cline { 2 - 4 } & Prob. & $0,004^{* *}$ & \\
\hline
\end{tabular}

$\mathrm{DDL}=$ degré de liberté, $\mathrm{RD}=$ Déviance résiduelle, Prob. = Probabilité, ${ }^{\mathrm{ns}}$ non significatif, *significatif au seuil $95 \%, * *$ significatif au seuil $99 \%$ 
Tableau 2. Productivités annuelles (moyenne et coefficient de variation en \%) de fruits et de semences viables par arbre des quatre espèces selon les classes de diamètre

\begin{tabular}{|c|c|c|c|c|c|}
\hline \multirow{2}{*}{ Espèce } & Classes de & \multicolumn{2}{|c|}{ Fruits } & \multicolumn{2}{c|}{ Graines } \\
\cline { 2 - 6 } & diamètre & $\mathrm{m}$ & $\mathrm{cv}(\%)$ & $\mathrm{cv}(\%)$ & $\mathrm{cv}(\%)$ \\
\hline \multirow{4}{*}{ A. paniculata } & $10-20$ & - & - & - & - \\
\cline { 2 - 6 } & $20-30$ & - & - & - & - \\
\cline { 2 - 6 } & $30-40$ & - & - & - & - \\
\cline { 2 - 6 } & $\geq 40$ & 59,00 & 14,38 & 1182,00 & 8,26 \\
\hline \multirow{5}{*}{ D. mespiliformis } & $10-20$ & 7882,00 & 100,95 & 14472,00 & 50,14 \\
\cline { 2 - 6 } & $20-30$ & 9421,00 & 46,47 & 355289,00 & 88,60 \\
\cline { 2 - 6 } & $30-40$ & 13663,00 & 84,45 & 208636,00 & 78,05 \\
\cline { 2 - 6 } & $\geq 40$ & 10182,00 & 66,34 & 510420,00 & 117,36 \\
\hline \multirow{5}{*}{ K. africana } & $10-20$ & 0,88 & 240,02 & 346,75 & 222,90 \\
\cline { 2 - 6 } & $20-30$ & 5,86 & 116,00 & 3458,29 & 108,43 \\
\cline { 2 - 6 } & $30-40$ & 9,13 & 112,09 & 2857,38 & 117,52 \\
\cline { 2 - 6 } & $\geq 40$ & 15,00 & 133,00 & 5774,11 & 168,03 \\
\hline & & & & & 33,08 \\
\hline \multirow{5}{*}{ V. doniana } & $10-20$ & 1546,50 & 31,52 & 1892,30 & 83,94 \\
\cline { 2 - 6 } & $20-30$ & 3066,00 & 83,72 & 3611,30 & 2,03 \\
\cline { 2 - 6 } & $30-40$ & 3565,80 & 80,52 & 4160,50 & 80,01 \\
\cline { 2 - 6 } & $\geq 40$ & 8932,00 & 2,03 & 10340,50 & \\
\end{tabular}

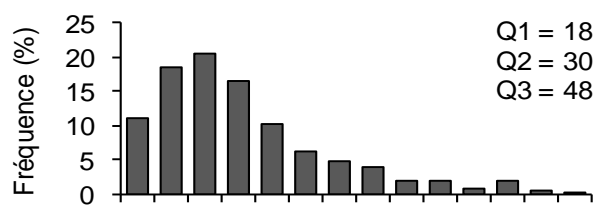

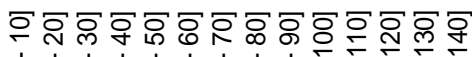

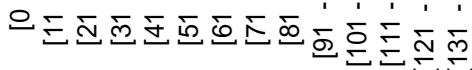

(a) Nombre de graines par fruit

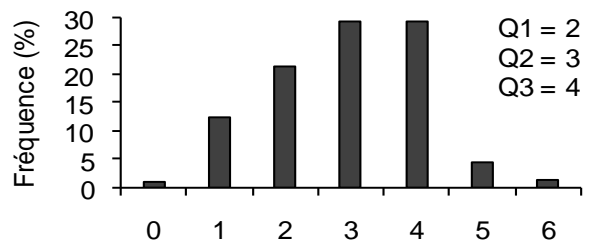

(b)

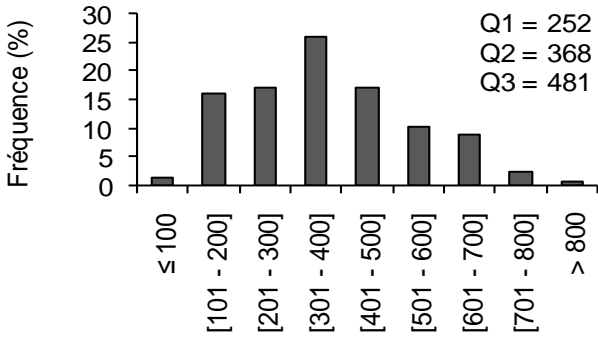

(c) Nombre de graines par fruit

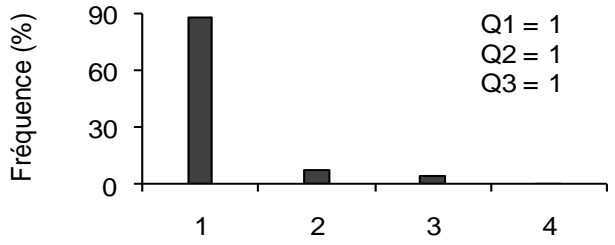

Nombre de graines par fruit

Figure 2. Distribution du nombre de graines par fruit de A. paniculata (a), D. mespiliformis

(b), K. africana (c) et V. doniana (d)- Q1 ; Q2 et Q3 sont respectivement les premier, deuxième et troisième quartiles. 


\section{Discussion}

La conservation d'une espèce végétale ne peut être assurée que par sa capacité de production fruitière et sa capacité de régénération. La présente étude a évalué l'influence de la classe de diamètre de quatre espèces ligneuses sur leur production annuelle de fruits et de semences viables en zone soudanienne au Bénin. Les résultats ont révélé que la classe de diamètre influence d'une part la production de fruits de $K$. africana et $V$. doniana, et d'autre part la production de semences viables de A. paniculata, D. mespiliformis et de $V$. doniana. Ainsi, la capacité de fructification et de régénération naturelle des espèces étudiées suivant les classes de diamètre varie d'une espèce à une autre.

Parmi les espèces étudiées, D. mespiliformis, A. paniculata et $K$. africana ne sont rencontrées que dans un seul type d'habitat (forêt dense). Ce type d'habitat est confirmé pour D. mespiliformis par Aubréville (1950), Arbonnier (2000) et Kouyaté (2005); et pour K. africana par Rønne et Jøker (2005). Néanmoins, la présence de $K$. africana a été signalée dans les agglomérations au Bénin (Akoègninou et al., 2006). Quant à $D$. mespiliformis, la consommation de ses fruits pendant la période de soudure a été signalée dans plusieurs régions de l'Afrique tropicale (Kouyaté 2005). En plus de la forêt dense, les individus de $V$. doniana sont également rencontrés dans différents types de formations végétales. Orwa et al. (2009) et Hounkpèvi et al. (2016) ont rapport que $V$. doniana était présente dans des habitats variés allant de la forêt à la savane.

La production fruitière suivant les classes de diamètre était significativement différente pour les espèces $K$. africana et $V$. doniana. Ainsi, la production fruitière croit au fur et à mesure que le diamètre de ces deux espèces augmente. Les mêmes observations ont été faites sur les espèces telles que Adansonia digitata L. en Afrique de l'Ouest (Schumann et al., 2010) au Sénégal (Sanogo et al., 2015) et au Bénin (Assogbadjo et al., 2005), Swietenia macrophylla au Mexique (Snook et al., 2005) et Detarium microcarpum Guil. \& Perr au Mali (Kouyaté et al., 2006). Cette influence des classes de diamètre pourrait être justifiée par le fait que les espèces à gros diamètre ont une tendance à avoir plus de branchage que celles de petit diamètre comme l'ont rapporté Assogbadjoet al. (2005) et Kouyaté et al. (2006). Cette corrélation entre la production fruitière et le diamètre permet donc de dire que pour les espèces, les arbres bons producteurs sont des arbres de gros diamètre (Sanogo et al., 2015). Par contre, au Burkina Faso, Guira et Zongo (2002) ont trouvé une corrélation négative entre le diamètre et la production de Vitellaria paradoxa Gaertn. indiquant que les arbres bons producteurs sont de petite taille. Le diamètre des arbres serait donc très utile pour la conservation des espèces spécialement pour les programmes d'amélioration génétique en vue de l'augmentation de leur productivité. 
Les paramètres morphologiques identifiés dans la présente étude comme facteurs influençant la production fruitière des espèces étudiées peuvent être pris en considération pour l'établissement des modèles prédictifs d'estimation ou de quantification des niveaux de productions des fruits et des graines. En effet, de tels modèles ont été établis pour d'autres espèces forestières à usages multiples (Assogbadjo et al., 2005; Kouyaté et al., 2006; Ouédraogo et al., 2014), et ont permis l'estimation plus aisée du potentiel productif de celles-ci. La reconnaissance du potentiel économique des quatre espèces étudiées pourrait augmenter l'intérêt pour leur conservation comme suggéré pour B. costatum (Ouédraogo et al., 2014). Il faudrait aussi sensibiliser les populations locales à la domestication de ces espèces à leur plantation.

\section{Conclusion}

La présente étude a évalué l'influence des classes de diamètre de quatre espèces ligneuses ( $K$. africana, $V$. doniana, A. paniculata et $D$. mespiliformis) sur leur production annuelle de fruits et de semences viables en zone soudanienne au Bénin. Les résultats ont révélé que la classe de diamètre influence d'une part la production de fruits de $K$. africana et $V$. doniana, et d'autre part la production de semences viables de $A$. paniculata, $D$. mespiliformis et de $V$. doniana. Les paramètres morphologiques influençant la productivité des espèces étudiées qui ont été identifiés pourraient être très utiles pour l'amélioration génétique de celles-ci avec pour objectif l'augmentation de leur productivité de fruits et de semences viables. Pour des perspectives futures de recherches, il serait très opportun de développer des méthodes d'estimation du potentiel économique de ces espèces. Il faudrait aussi sensibiliser les populations locales à la plantation de ces espèces et à des méthodes de récolte moins destructives.

\section{References:}

1. Achigan Dako, E.G., Pasquini M.W., Assogba Komlan, F., N'danikou S., Yédomonhan H., Dansi A., Ambrose Oji B. (2010). Traditional vegetables in Benin. Institut National des Recherches Agricoles du Bénin. Imprimeries du CENAP, Cotonou.

2. Akoègninou, A., van der Burg W.J. et van der Maesen L.J.G., 2006 : Flore analytique du Bénin. Cotonou \& Wageningen, Backuys Publishers, $1064 \mathrm{p}$.

3. Arbonnier, M. (2000). Arbres, arbustes et lianes des zones sèches d'Afrique de l'Ouest. CIRAD. MNHN. UICN. Montpellier. France. $541 \mathrm{p}$. 
4. Arbonnier, M. (2008). Faut-il tolérer des feux dans les parcs nationaux d'altitude en Afrique centrale? Bois et forêts des tropiques, 296, pp. 39-43.

5. Asase, A., Oteng-Yeboah, A.A., Odamtten, G.T., Simmonds, M.S.J., 2005. Ethnobotanical study of some Ghanaian anti-malarial plants. Journal of Ethnopharmacology 99, pp. 273-279.

6. Assogbadjo, A.E., Sinsin, B., and Van Damme, P. (2005). Caractères morphologiques et production des capsules de baobab (Adansonia digitata L.) au Bénin. Fruits 60, pp. 327-340.

7. Belem, B., Nacoulma, B.M.I., Gbangou, R., Kambou, S., Hansen, H.H., Gausset, Q., Lund, S., Raebild, A., Lompo, D., Ouédraogo, M., Theilade, I. \& Boussim, J. I.(2007). Use of Non Wood Forest Products by local people bordering the "Parc National Kaboré Tambi”, Burkina Faso. J. Transdiscipli. Environ. Stud. 6(1), pp. 1-21.

8. Cunningham, A.B. (2001). Applied ethnobotany: people, wild plant use and conservation. Earthscan.

9. Hounkpèvi, A., Tosso, F., Gbèmavo, D.S.J.C., Kouassi, E.K., Koné, D., Glèlè Kakaï, R. (2016). Climate and potential habitat suitability for cultivation and in situ conservation of the black plum (Vitex doniana Sweet) in Benin, West Africa. International Journal of Agronomy and Agricultural Research, 8(4), pp. 67-80.

10. Hutchinson, J.\& Dalziel, J.M. (1963). Rubiaceae. Flora of West Tropical Africa, ed, 2, 213.

11. Igoli, J.O., Ogaji, O.G., Tor-Anyiin T.A., Igoli P.N. (2005). Traditional medicine practice amongst the Igede people of Nigeria. Part II. African J Trad Compl Altern Med., 2, pp. 134-152.

12. Irvine, F. R. (1961). Woody plants of Ghana, with special reference to their uses, Oxford University Press, London, United Kingdom, pp. 736-740.

13. Kouyaté, A. M., Van Damme, P., Diawara M. (2006). Évaluation de la production en fruits de Detarium microcarpum Guil. \& Perr au Mali. Fruits 61, pp. 267-272.

14. Kouyaté, A.M. (2005). Aspects ethnobotaniques et étude de la variabilité morphologique, biochimique et phénologique de Detarium Microcarpum Guill. \& Perr. au Mali. Thèse de doctorat unique : Université de Gent, Belgique.

15. Ky, K. J. M. (2008). Vitex doniana sweet. In: Louppe D, Oteng Amoako AA, Brink M (eds) Prota: Timbers/Bois d'oeuvre, vol 7(1). Backhuys Publishers, Leiden, pp 578-581.

16. Lamien, N., Tigabu, M., Guinko, S., \& Oden, P.C. (2007). Variations in dendrometric and fruiting characters of Vitellaria paradoxa 
populations and multivariate models for estimation of fruit yield. Agroforestry Systems, 69(1), pp. 1-11.

17. Maisiri, M. and Gundidza, M. 1999. The effects ofcrude extracts of Kigelia africana andAloe excelsa on deep wound healing,University of Zimbabwe, Harare, Departmentof Pharmacy, pp. 91-94.

18. Nakazawa,M. (2014). fmsb: Functions for medical statistics book with some demographic data. $\mathrm{R}$ package version 0.4.3. http://CRAN.R-project.org/package $=$ fmsb

19. Nacoulma, B. M. I. (2012). Dynamique et stratégies de conservation de la végétation et de la phytodiversité du complexe écologique du parc national du W du Burkina Faso (Doctoral dissertation, Dissertation, University of Ouagadougou).

20. Orwa, C., Mutua, A., Kindt, R., Jamnadass, R., Simons, A. (2009). Agroforestree Database: a tree reference and selection guide version 4.0. http://www.worldagroforestry.org/af/treedb/

21. Ouédraogo, I., Nacoulma, B.M.I., Ouédraogo, O., Hahn, K. \& Thiombiano, A. (2014). Productivité et valeur économique des calices de Bombax costatum Pellegr. \& Vuillet en zone soudanienne du Burkina Faso. Bois For. Trop. 319(1), pp. 31-41.

22. Ouôba, P., Boussim, J., \& Guinko, S. (2006). Le potentiel fruitier de la forêt classée de Niangoloko au Burkina Faso. Fruits, 61(1), pp. 7181.

23. Pooley, E. (1993). The complete guide to trees of Natal, Zululand and Transkei. Natal FloraPublications Trust, 1993, pp. 22-24.

24. R Core developmental Team (2013). R: A language and environment for statistical computing. R Foundation for Statistical Computing, Vienna, Austria. ISBN3-900051-07-0, URL http://www.Rproject.org/.

25. Rønne, C., \& Jøker, D. (2005). Kigelia africana (Lam.) Benth. Seed Leaflet, (108), 1-2.

26. Sai, F.L. (1997). Fruit and Vegetables in West Africa; Food and Agriculture Organisation of the United Nation, Rome. 5 - 6.

27. Saini, S., Kaur, H., Verma, B., Ripudaman, and Singh, S. K. (2009). Kigelia africana (Lam.) Benth.-An overview. Nat. Prod. Radiance8: 190-197.

28. Sanogo, D., Badji, M., Diop, M., Samb, C.O., Tamba, A. \& Gassama, Y. K. (2015). Évaluation de la production en fruits de peuplements naturels de Baobab (Adansonia digitata L.) dans deux zones climatiques au Sénégal. Journal of Applied Biosciences, 85, 78387847.

29. Schumann, K., Wittig, R., Thiombiano, A., Becker, U., Hahn, K. (2010). Impact of land-use type and bark- and leaf-harvesting on 
population structure and fruit production of the baobab tree (Adansonia digitata L.) in a semi-arid savanna, West Africa. Forest Ecology and Management, 260 (11): 2035-2044.

30. Snook, L.K., Camara-Cabrales, L., and Kelty, M.J. (2005). Six years of fruit production by mahogany trees (Swietenia macrophylla King): patterns of variation and implications for sustainability. Forest Ecology and Management, 206, pp. 221-235.

31. Sop, T.K., Oldeland, J., Bognounou, F., Schmiedel, U. \& Thiombiano, A. (2012). Ethnobotanical knowledge and valuation of woody plants species: a comparative analysis of three ethnic groups from the sub-Sahel of Burkina Faso. Environ. Dev. Sustain., 14(5), pp. 627-649.

32. Venables, W. N. \& Ripley, B. D. (2002). Modern Applied Statistics with S. Fourth Edition. Springer, New York. ISBN 0-387-954570Turnbull L.A., Crawley M.J. \& Rees M., 2000. Are plant populations seed-limited? A review of seed sowing experiments. Oikos, 88, pp. 225-238.

33. Vodouhê, F.G., Coulibaly, O., Greene, C., and Sinsin, B. (2009). Estimating the Local Value of Non-Timber Forest Products to Pendjari Biosphere Reserve Dwellers in Benin. Economic Botany, 63(4), pp. 397-412.

34. Vunchi, M.A., Umar, A.N., King, M.A., Liman, A.A., Jeremiah, G. and Aigbe, C.O. (2011). Proximate, Vitamins and Mineral Composition of Vitex doniana (black plum) Fruit Pulp. Nigerian Journal of Basic and Applied Science, 19(1), pp.97-101.

35. Zizka, A., A. Thiombiano, S. Dressler, B. Nacoulma, A.Ouédraogo, I. Ouédraogo, O.Ouédraogo, G. Zizka, K.Hahn \& M. Schmidt (2015). Traditional plant use in BurkinaFaso (West Africa): a national-scale analysis with focus on traditionalmedicine. J. Ethnobiol. Ethnomed. 11: 9 . 